\title{
Liponeurocytoma of the Cerebellum with Myoid Features
}

\author{
Chbani Laila ${ }^{*}$, Badioui Ikram¹, Znati Kaoutar', Benzagmout Mohamed ${ }^{2}$, Maaroufi Mustapha ${ }^{3}$, \\ Amarti Afaf ${ }^{1}$
}

${ }^{1}$ Department of Pathology, Hassan II Teaching Hospital, Fez, Morocco; ${ }^{2}$ Department of Neurosurgery, Hassan II Teaching Hospital, Fez, Morocco; ${ }^{3}$ Department of Radiology, Hassan II Teaching Hospital, Fez, Morocco.

Email: *chbanil@yahoo.fr

Received February $10^{\text {th }}, 2012$; revised February $27^{\text {th }}, 2012$; accepted March $28^{\text {th }}$, 2012

\begin{abstract}
We report an extremely rare tumor presenting with myoid features in the left cerebellum lobe in a 62-year-old man. This tumor consisted of medium to large round cells with focal lipomatous and myoid differentiation. Immunohistochemically, the tumor cells expressed synaptophysin, GFAP (glial fibrillary acidic protein) and focally desmin. From these findings, we concluded that this tumor was a liponeurocytoma with myoid features. To our knowledge, this is the second report of liponeurocytoma with myoid differentiation in the cerebellum.
\end{abstract}

Keywords: Liponeurocytoma; Cerebellum; Myoid Differentiation; Immunohistochemistry

\section{Introduction}

Cerebellar liponeurocytoma is morphologically composed of uniform round cells with neuronal differentiation and lipomatous features. It typically affects adults and has a favourable prognosis. Initially described as a lipomatous medulloblastoma [1], the World Health Organisation Working group [2] proposes the term "cerebellar liponeurocytoma" primarily to distinguish this entity from medulloblastoma and to avoid over treatment of this benign tumor.

Here, we report the second case in our knowledge in the literature data of cerebellar liponeurocytoma with myoid features and discuss the histogenesis of this rare tumor.

\section{Case Report}

The patient was a 62-year-old man who presented with a 4-month history of gradually headache, vomiting and decreased visual acuity. His past medical and family history were unremarkable. Neurological examination revealed cerebellar syndrome. Magnetic resonance imaging (MRI) showed a relatively well-defined, poorly enhancing, round lesion in the left cerebellar hemisphere hyperintense in T2-Weighted, causing moderate mass effect (Figure 1). At surgery, a $5.7 \mathrm{~cm} \times 4.7 \mathrm{~cm}$, well circumscribed mass, located in the cerebellar left hemisphere was removed.

Ten percent formalin-fixed specimens were paraffin-

"Corresponding author. embedded, cut at $3 \mu \mathrm{m}$, and stained with hematoxylineosin-saffron.

Microscopically, the tumor was composed of small round cells to polygonal with eosinophilic or clear cytoplasm resembling oligodendrocytes. In some areas, the tumor cells were mixed with lipomatous cells (Figure 2) and myocyte-like cells. The tumor shows no mitotic figures.

An immunohistochemical study was performed. After heat-induced epitope retrieval, dewaxed $3 \mu \mathrm{m}$ sections were incubated with the following prediluted antibodies: synaptophysin (Cell Marque, Rabbit polyclonal), GFAP (glial fibrillary acidic protein) (Cell Marque, Clone G-A5, Mouse monoclonal), Mib1 (Cell Marque, Clone sp6, Rabbit monoclonal) and desmin (Cell Marque, clone D33, Mouse monoclonal). Revelation was performed after a streptavidin-biotin-peroxidase technique with diaminobenzidine.

Tumour cells expressed synaptophysin, GFAP and focally desmin (Figure 3 ). The Mib 1 antibody stained less than $1 \%$ of tumour cell nuclei.

The tumor was classified as a cerebellar liponeurocytoma with myoid differentiation grade II according to the WHO grading [2].

His postoperative period was uneventful with no evidence of tumour recurrence after 12 months.

\section{Discussion}

Liponeurocytoma is a rare cebellar neoplasm of adults that is histologically characterized by neuronal different- 


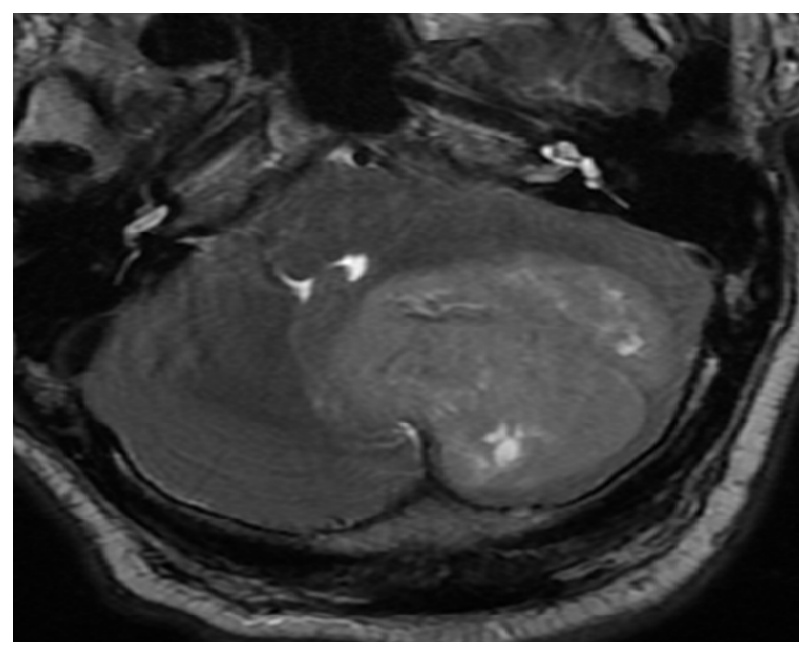

Figure 1. T2-Weighted MRI of cerebellar tumour.

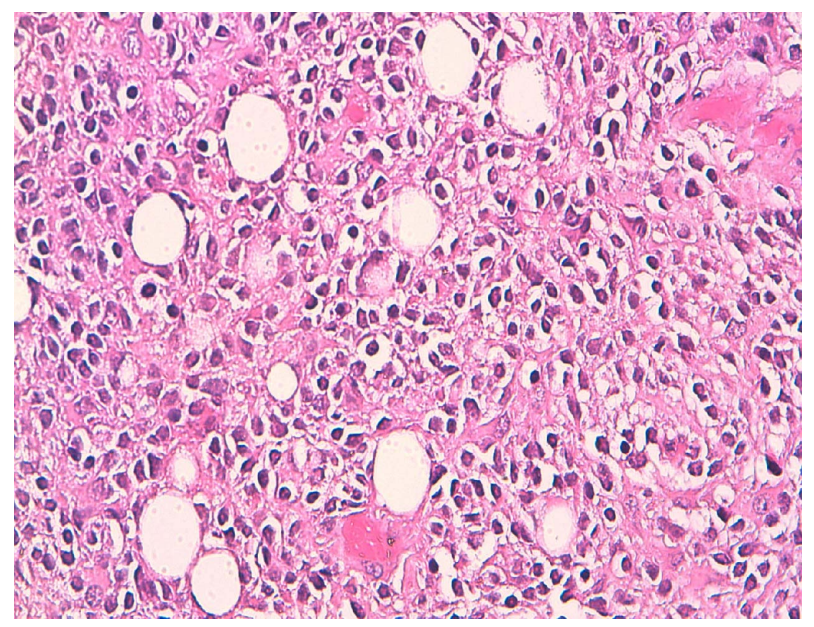

Figure 2. Isomorphic small cells with focal lipomatous differentiation, $H \& E^{*} 250$.

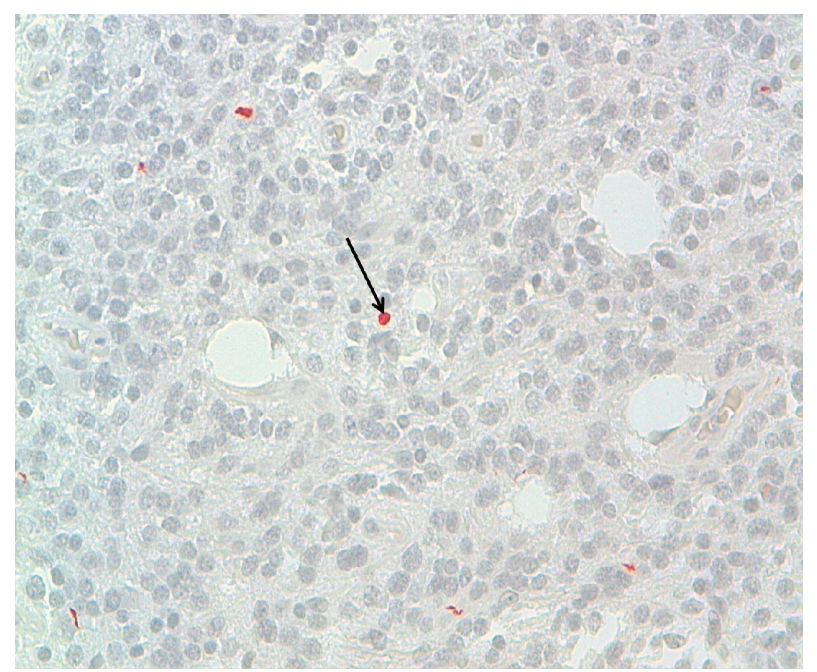

Figure 3. Immunoreactivity with anti-desmin, DAB $\times 100$. Positivity of some tumor cells, indicating an myogenic differentiation (Arrows). iation with focal lipidization and low proliferative potential.

Since the first report [1] by Bechtel in 1978 as a mixed mesenchymal and neuroectodermal tumor of the cerebellum, several terms "medullocytoma” [2], "lipomatous glioneurocytoma" [3] and "lipidized mature neuroectodermal tumor of the cerebellum” [4] have been proposed as to emphasize its resemblance to central neurocytoma.

In 2000 [2], the WHO classification of tumours of the central nervous system proposed the term "cerebellar liponeurocytoma” to distinguish this tumor from medulloblastoma with lipidized cells because the outcome and to avoid aggressive treatment to this benign tumor.

This tumor classically manifests at the fifth decades (mean 50 years) [5] without significant gender predilection. This is in strong contrast with the age distribution of medulloblastoma, which commonly occurs in the pediatric age group.

The clinical features are not specific.

This tumour may be situated within the cerebellar vermis or the hemispheres.

Histologically, the cerebellar liponeurocytoma features a monotonous pattern of round cells with focal lipomatous differentiation, characterized by lipidized cells ressembling mature adipose tissue [6]. Tumour cells have round and small nuclei and often show a clear cytoplasm resembling neoplastic oligodendrocytes.

The round and lipidized cells expressed neuronal markers and focally GFAP in the majority of cases. To the best of our knowledge, only a single case of liponeurocytoma with myoid differentiation has been previously reported by Gonzalez et al. [4] (Table 1).

The Mib1 (KI67) proliferation index was between 1\% and $6 \%$ in the majority of cases reported in the literature data [7].

The histogenesis of cerebellar liponeurocytoma has remained unclear. The cell origin is most likely a precursor cell with preferential neuronal differentiation but a capacity for divergent, astrocytic and myogenic differentiation.

An origin from the external granular layer of the cerebellum cannot be ruled out according to Burhen et al. [8].

The most important differential diagnosis is the medulloblastoma with lipidized cells. However the high index proliferation and young age of the patient in medulloblastoma can help to distinguish between these two neoplasms.

A complete surgical resection is recommended as the initial treatment but there is no consensus regarding the treatment of this rare tumour specifically adjuvant chemotherapy or radiotherapy.

The adjuvant radiotherapy is indicated in some cases with recurrence [7].

The prognosis of liponeurocytoma is favourable and 
Table 1. Liponeurocytoma of the cerebellum with myoid differentiation (review).

\begin{tabular}{|c|c|c|c|c|c|c|}
\hline Authors/year & Age/Sex & Location & MRI & Immunostaining & $\begin{array}{l}\text { Adjuvant } \\
\text { therapy }\end{array}$ & Follow up \\
\hline $\begin{array}{c}\text { Gonzales-Campora } \\
\text { et al. } 1998\end{array}$ & $67 \mathrm{~F}$ & $\begin{array}{l}\text { Vermis and right } \\
\text { cerebellar hemisphere }\end{array}$ & $\begin{array}{l}\text { Inhomogeneous mass with } \\
\text { high } \mathrm{T} 1 \text { signal intensity }\end{array}$ & $\begin{array}{l}\text { Small cells: SYN+GFAP+ and } \\
\text { S-100+/- } \\
\text { Adipocytes/SYN, GFAP, S-100+ } \\
\text { Muscle cells: Desm+ }\end{array}$ & - & $\begin{array}{c}\text { Dead, } \\
\text { post-operative }\end{array}$ \\
\hline Chbani et al. 2012 & $62 \mathrm{M}$ & $\begin{array}{l}\text { Left cerebellar } \\
\text { hemisphere }\end{array}$ & $\begin{array}{l}\text { Round lesion hyperintense } \\
\text { in T2-Weighted }\end{array}$ & $\begin{array}{l}\text { Small cells: SYN+GFAP+ and } \\
\text { S-100+/- } \\
\text { Adipocytes/SYN, GFAP, S-100+ } \\
\text { Muscle cells: Desm+ }\end{array}$ & - & $\begin{array}{l}\text { One year, without } \\
\text { recurrence }\end{array}$ \\
\hline
\end{tabular}

the 5-year survival rate was $48 \%$ [7].

According to Horstmann et al. [7], 62\% of patients developed a recurrence after periods ranging from 1 to 12 years.

Histopathological features predicting recurrence have not been identified.

\section{Conclusion}

The cerebellar liponeurocytoma is a histopathologically and genetically distinct entity. The distinction between liponeurocytoma and medulloblastoma is very important since the medulloblastoma require adjuvant radio/chemotherapy.

\section{REFERENCES}

[1] J. T. Bechtel, J. M. Patton and Y. Takei, "Mixed Mesenchymal and Neuroectodermal Tumor of the Cerebellum," Acta Neuropathologica, Vol. 41, No. 3, 1978, pp. 261263. doi:10.1007/BF00690447

[2] D. N. Louis, H. Ohgaki, O. D. Wiestler and W. K. Cavenee, "Pathology and Genetics of Tumours of the Nervous System,” In: P. Kleihues and L. H. Sobin, Eds., World Health Organization Classification of Tumours, International Agency for Research on Cancer, Lyon, 2007, p. 110.

[3] C. H. Alleyne Jr., S. Hunter, J. J. Olson and D. L. Barrow,
"Lipomatous Glioneurocytoma of the Posterior Fossa with Divergent Differentiation: Case Report,” Neurosurgery, Vol. 42, No. 3, 1998, pp. 639-643. doi:10.1097/00006123-199803000-00035

[4] R. Gonzalez-Campora and R. O. Weller, "Lipidized Mature Neuroectodermal Tumor of the Cerebellum with Myoid Differentiation," Neuropathology and Applied Neurobiology, Vol. 24, No. 5, 1998, pp. 397-402. doi:10.1046/j.1365-2990.1998.00139.x

[5] N. Montagna, L. C. Vaz and M. A. Astro, "Meduloblastoma Cerebelar Em Adulto com Areas Lipomatosas," Journal Brasileiro de Patologia e Medicina Laboratorial, Vol. 35, No. 49, 1999.

[6] B. K. Owler, J. M. Makeham, M. Shingde and M. Besser, “Cerebellar Liponeurocytoma," Journal of Clinical Neuroscience, Vol. 12, No. 3, 2005, pp. 326-329. doi:10.1016/j.jocn.2004.05.017

[7] S. Horstmann, A. Perry, G. Reifenberger, et al., "Genetic and Expression Profiles of Cerebellar Liponeurocytomas,” Brain Pathology, Vol. 14, No. 3, pp. 281-289.

[8] J. Buhren, A. H. Christoph, R. Buslei, S. Albrecht, O. D. Wiestler and T. Pietsch, "Expression of the Neurotrophin Receptor p75NTR in Medulloblastomas Is Correlated with Distinct Histological and Clinical Features: Evidence for a Medulloblastoma Subtype Derived from the External Granule Cell Layer,” Journal of Neuropathology Experimental Neurology, Vol. 59, No. 3, 2000, pp. 229240. 